\title{
Structural lesion to ribonuclease A caused by reductive stress: Assessment by Raman spectroscopy
}

\author{
Armida Torreggiani \\ Consiglio Nazionale delle Ricerche, Istituto ISOF, via P. Gobetti 101, 40129 Bologna, Italy \\ Tel.: +390516399821; Fax: +39051 6399844; E-mail: torreggiani@isof.cnr.it
}

\begin{abstract}
The damages induced by reductive radical stress on bovine pancreatic ribonuclease A (RNAse A) were investigated by Raman spectroscopy. Gamma-irradiation was used to simulate the endogenous formation of reductive species, in particular ${ }^{-} \mathrm{H}$ atom that is a simple one-electron equivalent reducing agent. Specific damages occur at sensitive amino acid sites, selectively, rather than indiscriminately, leading to the structure modification of the protein. Sulfur-containing residues (Met and Cys) and aromatic residues are appreciably attacked. In particular, extensive changes in the disulfide bridge conformations are induced as well as conformational changes of the protein secondary structure; a gradual conversion of $\alpha$-helical to pleated-sheet geometry was evidenced, indicating a higher ability of reducing radicals in denaturing the protein structure compared with that of oxidizing radical species.
\end{abstract}

Keywords: Bovine pancreatic ribonuclease A, Raman spectroscopy, $\gamma$-irradiation, radical damage

\section{Introduction}

Protein damage caused by free radicals makes part of the aetiology of several diseases and the aging processes. Exposure of proteins to free radicals may cause structural modifications of primary, secondary and tertiary assembly; consequently, the activity of enzymes, receptors and membrane transporters can be greatly affected $[1,2]$. In this respect, the fate of radical species derived from protein damage has been subject of many investigations, although a clear picture of the degradation paths and their influence on the disease aetiology is far from being achieved. The most studied intermediates known to cause protein damage are reactive oxygen species (ROS) and in particular ${ }^{\bullet} \mathrm{OH}$ radicals [3], whereas the reductive stress has been less considered.

In a previous paper both the functional and structural changes of bovine pancreatic ribonuclease A (RNAse A) have been analysed under mainly oxidative radical conditions $\left({ }^{\bullet} \mathrm{OH} 90 \%\right.$ and $\left.\mathrm{H}^{\bullet} 10 \%\right)$ and a stronger reactivity involving in particular sulfur-containing residues and aromatic residues has been shown [4]. Since recently the reductive stress has been placed in the context of a biological damage, constituting the basis of tandem-lipid damage the separate contribution of a reducing species such as $\mathrm{H}^{\bullet}$ to the changes produced in RNAse was investigated more in detail in the present work $[5,6]$. Neutral hydrogen atom is a simple one-electron equivalent reducing agent and its reactions are of great interest since they can shed light on basic problems of one-electron equivalent reaction kinetics.

RNAse A is a small protein (MW 13700) consisting of one polypeptide chain cross-linked by four disulfide bonds and a total of 124 amino acid residues of which six are Tyr, four Hys and four Met; 
Trp and free Cys are not present. X-ray crystallographic analysis of RNAse has evidenced the presence of a nine antiparallel $\beta$-sheet and three helices ( $1 \mathrm{kf} 5$ in PDB, $1.15 \AA$ resolution). The degradation of RNAse A due to radical exposure was followed by Raman spectroscopy and the conditions of an endogenous radical stress were mimicked by using $\gamma$-radiolysis as source of free radicals. To identify more specifically the action of reductive radicals, irradiations were performed under various environmental conditions to isolate the individual radicals.

\section{Experimental}

Bovine pancreatic ribonuclease A (RNAse A, EC. 3.1.27.5) was purchased from Sigma. RNAse A solutions were freshly prepared in tri-distilled water $(0.6 \mathrm{mg} / \mathrm{ml})$ and phosphate buffer $(10 \mathrm{mM})$. All solutions contained $t$ - $\mathrm{BuOH}$ to avoid the effects of the oxidizing ${ }^{\circ} \mathrm{OH}$ radicals. Aliquots of the solution were transferred in different vials and saturated with $\mathrm{N}_{2} \mathrm{O}$ or Ar. One of the vials was directly lyophilised, whereas the other vials were irradiated at different times and then lyophilised.

Continuous radiolysis was performed on RNAse A aqueous solutions by using a ${ }^{60} \mathrm{Co}-\mathrm{Gammacell}$ at the dose rate of $11 \mathrm{~Gy} / \mathrm{min}$. The reaction sequence starts from primary water reactive species $\left(\mathrm{e}_{\mathrm{aq}}{ }^{-}\right.$, $\bullet \mathrm{OH}$ and $\mathrm{H}^{\bullet}$ ) obtained by $\gamma$-radiolysis of aqueous solutions or suspensions (Eq. (1)). By saturating the samples by $\mathrm{N}_{2} \mathrm{O}$ prior to irradiation $(\approx 0.025 \mathrm{M})$, solvated electrons are converted into hydroxyl radicals (Eq. (2), $\left.k_{2}=9.1 \times 10^{9} \mathrm{M}^{-1} \mathrm{~s}^{-1}\right) . t$-BuOH was employed as an efficient scavenger for hydroxy radicals (Eq. (3), $k_{3}=6.0 \times 10^{8} \mathrm{M}^{-1} \mathrm{~s}^{-1}$ ). Oxygen-free solutions were obtained by Ar saturation where hydrated electrons may be converted into $\mathrm{H}^{\bullet}$ atoms in the presence of $10 \mathrm{mM} \mathrm{H}_{2} \mathrm{PO}_{4}{ }^{-}$solutions depending on the $\mathrm{pH}$ (Eq. (4), $k_{4}=1.5 \times 10^{7} \mathrm{M}^{-1} \mathrm{~s}^{-1}$ ).

$$
\begin{aligned}
& \mathrm{H}_{2} \mathrm{O} \rightsquigarrow \mathrm{e}_{\mathrm{aq}}{ }^{-},{ }^{\bullet} \mathrm{OH},{ }^{\bullet} \mathrm{H}, \\
& \mathrm{e}_{\mathrm{aq}}{ }^{-}+\mathrm{N}_{2} \mathrm{O} \stackrel{\mathrm{H}_{2} \mathrm{O}}{\longrightarrow} \mathrm{N}_{2}+\mathrm{OH}^{-}+{ }^{\bullet} \mathrm{OH}, \\
& \bullet \mathrm{OH}+t-\mathrm{BuOH} \longrightarrow\left(\mathrm{CH}_{3}\right)_{2} \mathrm{C}(\mathrm{OH}) \mathrm{CH}_{2}^{\bullet} \mathrm{H}_{2} \mathrm{O}, \\
& \mathrm{e}_{\mathrm{aq}}{ }^{-}+\mathrm{H}_{2} \mathrm{PO}_{4}{ }^{-} \longrightarrow \cdot \stackrel{\mathrm{H}}{\longrightarrow}+\mathrm{HPO}_{4}{ }^{2-} .
\end{aligned}
$$

By taking into account the rate constant for the reaction of hydrated electron with RNAse A, which is reported to be $1 \times 10^{10} \mathrm{M}^{-1} \mathrm{~s}^{-1}$ at $\mathrm{pH} 7.0$ [7], $20 \%$ of the hydrated electrons are converted into $\mathrm{H}^{\bullet}$ atoms, of which only $\approx 70 \%$ will react with RNAse A because of the presence of $t$-BuOH. Thus, under these experimental conditions the formation yield of $\mathrm{H}^{\bullet}$ atom is increased of about $25 \%$ compared with that obtained in $\mathrm{N}_{2} \mathrm{O}$-saturated samples ( 0.83 and $0.6 \mu$ molj $^{-1}$, respectively).

Raman spectra were obtained on lyophilised samples by a Bruker IFS 66 spectrometer equipped with a FRA-106 Raman module and a cooled Ge-diode detector. The excitation source was a $\mathrm{Nd}^{3+}-\mathrm{YAG}$ laser (power at the sample $100 \mathrm{~mW}$ ). The curve fitting analysis was implemented by using the OPUS/IR version 5.0 program, which uses the Levenberg-Marquardt algorithm. The Raman component profiles were described as a linear combination of Lorenz and Gauss functions.

\section{Results and discussion}

To follow the changes in the protein structure and the microenvironment of amino acidic residues, resulting from the exposure to $\mathrm{H}^{\bullet}$ atoms, RNAse A was irradiated in $\mathrm{N}_{2} \mathrm{O}$-saturated aqueous solutions 


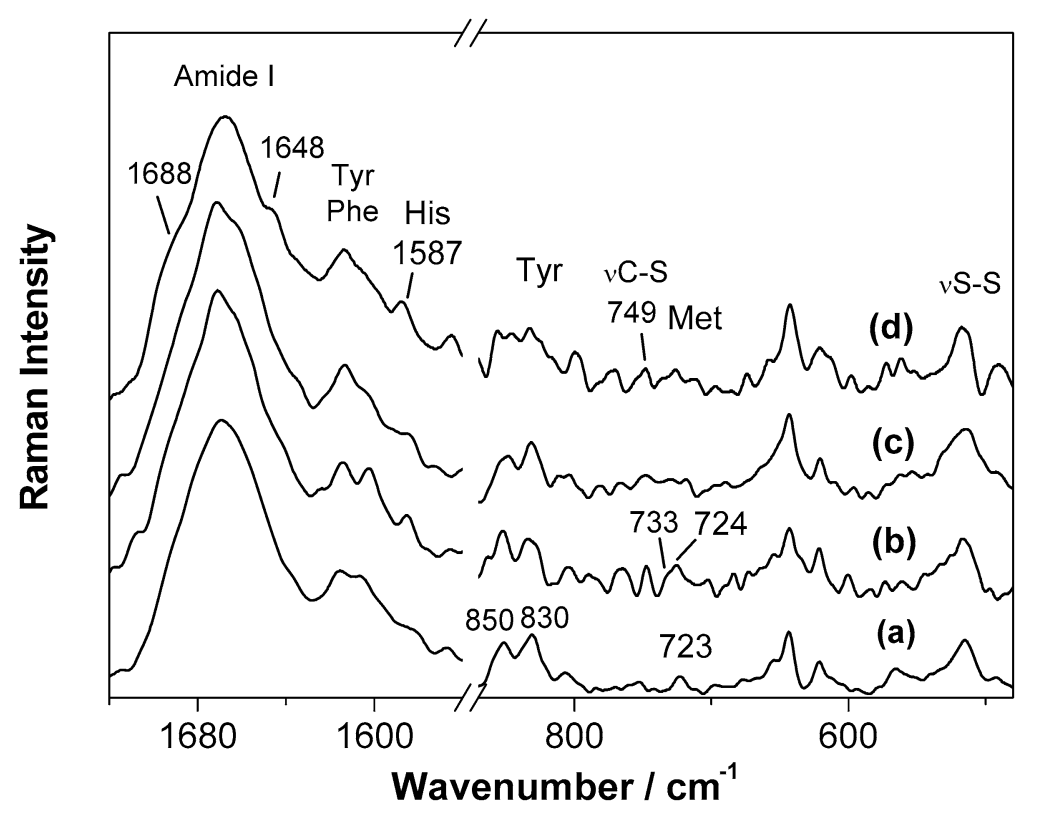

Fig. 1. The Raman spectra of native RNAse A (a) and $\gamma$-irradiated RNAse A at different doses: 23 (b), 61 (c) and 84 Gy (d).

containing $t$ - $\mathrm{BuOH}$ as ${ }^{\bullet} \mathrm{OH}$ scavenger; under these conditions $\mathrm{H}^{\bullet}$ atoms are the only reducing radical species. Figure 1(a) shows the Raman spectra of native and irradiated RNAse A with different doses (from 23 to $84 \mathrm{~Gy}$ ). From a qualitative examination of the spectra many differences were evident, in particular in the Amide I band, which is a conformational marker, and the bands due to Tyr, Met and disulfide bridges.

The evaluation of the conformational changes of RNAse A upon irradiation was obtained by the Amide I analysis by two methods, one based on a combination of X-ray and Raman data base and the other involving the deconvolution of the spectral range of interest into component bands [8,9]. A similar trend of conformational changes was obtained by both methods (Table 1). As a consequence of $\gamma$-irradiation, the $\beta$-sheet percentage sensitively increased whereas the $\alpha$-helix content decreased. The percentage of the disordered conformation remained virtually unchanged up to 61 Gy and slightly increased after the highest dose. By comparing these results with those estimated after oxidative radical attack, a similar trend of changes was evidenced as well as a less ability from ${ }^{\bullet} \mathrm{OH}$ in denaturating the protein with respect to ${ }^{\bullet} \mathrm{H}$ atom (Table 1). In fact, a significant modification in the $\alpha$-helix and $\beta$-sheet content was caused only by the lowest irradiation dose, whereas the ${ }^{\bullet} \mathrm{H}$ atom attack induced ever-increasing marked changes in the protein structure at higher doses. It worth noting that the secondary structure percentages of RNAse A before irradiation are slightly different in the two systems, indicating that the addition of the small amount of $t-\mathrm{BuOH}$ to the aqueous solution induces itself a slight conformational change in the protein structure.

Among the amino acid residues present in RNAse A, Tyr resulted to be among the most sensitive residues towards radical attack. This conclusion was drawn out from the analysis of the doublet at 850$830 \mathrm{~cm}^{-1}$ that depends on the state of the hydrogen bonding involving the $\mathrm{OH}$ group of Tyr [4]. This intensity ratio, average of all six Tyr residues, was about 0.8 in untreated RNAse $\mathrm{A} t$-BuOH-containing aqueous solution, indicating the presence of three or four "buried" Tyr (Fig. 1(a)). The $I_{850} / I_{830}$ ratio increased of $\approx 30 \%$ after 23 Gy irradiation, indicating that one or two Tyr residues are located in a more 
Table 1

Percentages of secondary structure of neat RNAse A and the irradiated RNAse A with different doses obtained by the analysis of the Amide I Raman region

\begin{tabular}{|c|c|c|c|c|c|c|c|c|c|}
\hline \multirow{2}{*}{$\begin{array}{l}\text { Dose } \\
\text { (Gy) }\end{array}$} & \multicolumn{3}{|c|}{$\alpha$-Helix $(\%)$} & \multicolumn{3}{|c|}{$\beta$-Sheet $(\%)$} & \multicolumn{3}{|c|}{ Random (\%) } \\
\hline & \multicolumn{2}{|c|}{$\bullet \mathrm{H}^{1}$} & \multirow{2}{*}{$\frac{{ }^{\bullet} \mathrm{OH},{ }^{\bullet} \mathrm{H}^{2}}{18^{*}}$} & \multicolumn{2}{|c|}{${ }^{\bullet} \mathrm{H}$} & \multirow{2}{*}{$\frac{{ }^{\bullet} \mathrm{OH},{ }^{\bullet} \mathrm{H}}{48^{*}}$} & \multicolumn{2}{|c|}{$\bullet^{\bullet} \mathrm{H}$} & \multirow{2}{*}{$\frac{{ }^{\bullet} \mathrm{OH},{ }^{\bullet} \mathrm{H}}{34^{*}}$} \\
\hline 0 & $14^{*}$ & $17^{* *}$ & & $52^{*}$ & $53^{* *}$ & & $34^{*}$ & $30^{* *}$ & \\
\hline 23 & 11 & 14 & 12 & 55 & 57 & 54 & 34 & 29 & 35 \\
\hline 61 & 8 & 9 & 12 & 57 & 60 & 54 & 35 & 31 & 35 \\
\hline 84 & 5 & 4 & 12 & 58 & 59 & 54 & 37 & 37 & 35 \\
\hline
\end{tabular}

${ }^{1} \mathrm{RNAse} \mathrm{A}$ in $\mathrm{N}_{2} \mathrm{O}$-saturated aqueous solutions containing $<2 \% \mathrm{v} / \mathrm{v} t$-BuOH. ${ }^{2} \mathrm{RNAse} \mathrm{A}$ in $\mathrm{N}_{2} \mathrm{O}$-saturated aqueous solutions. These percentages are reported in reference [4]. * Method reported in reference [8]; ${ }^{* *}$ Curve fitting method.

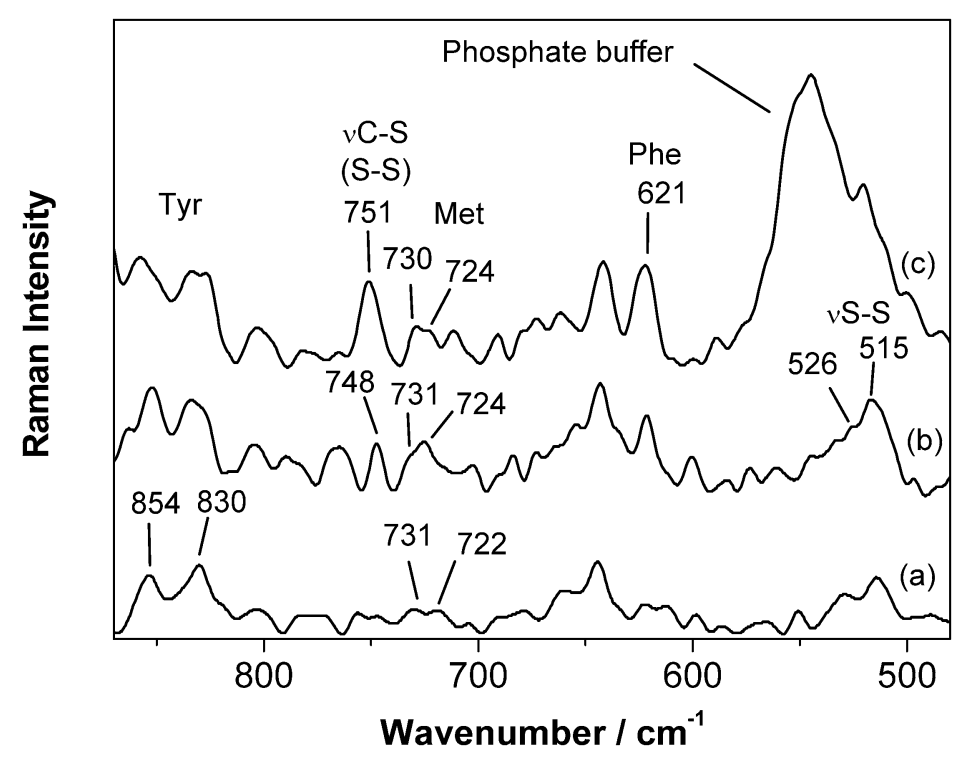

Fig. 2. The 900-450 $\mathrm{cm}^{-1}$ Raman region of low dose-irradiated RNAse A samples (23 Gy) obtained from: (a) $\mathrm{N}_{2} \mathrm{O}$-saturated RNAse A solution; (b) $\mathrm{N}_{2} \mathrm{O}$-saturated $t$-BuOH-containing RNAse A solution; (c) Ar-deaerated $t$-BuOH-containing RNAse A solution.

hydrophilic environment (Fig. 1(b)). The opposite behaviour has been observed by irradiating with the same dose the $\mathrm{N}_{2} \mathrm{O}$-saturated protein solution (Fig. 2(a)). Since similar conformational changes take place both in the case of the ${ }^{\bullet} \mathrm{OH}$ and ${ }^{\bullet} \mathrm{H}$ attack (Table 1), the different result can be mainly attributed to the different mechanism of action of the two reactive species. ${ }^{\circ} \mathrm{OH}$ radicals are more reactive and less selective than ${ }^{\bullet} \mathrm{H}$ and probably attack the enzyme surface. Of consequence, after the ${ }^{\bullet} \mathrm{OH}$ attack the more exposed Tyr, leading to $\mathrm{TyrO}^{\bullet}$ radicals and its subsequent pathway, would not be able to contribute to the doublet intensity. On the contrary, $\mathrm{H}^{\bullet}$ atoms probably attack the enzyme at a more restricted number of sites, perhaps away from the surface and also the far-reaching Tyr can be damaged. A confirm of this behaviour is given by the absence of aggregate formation after $\mathrm{H}^{\bullet}$ atom attack [5], whereas highly aggregated forms of the enzyme have been found under oxidative stress conditions $[6,10]$, since the $\mathrm{TyrO}^{\bullet}$ radicals give rise easier to inter-molecular reactions than intra-molecular ones. Since three Tyr "buried" can be probably identified with Tyr-25, -92 and -97 , the initial $\mathrm{H}^{\bullet}$ radical attack should take place on one or two of them. 
By increasing the dose up to $84 \mathrm{~Gy}$, the doublet bands broadened and the $I_{850} / I_{830}$ ratio decreased, analogously to that observed after the ${ }^{\bullet} \mathrm{OH}$ attack. This result, indicating a relevant decrease in the amount of the exposed Tyr residues, can be correlated with the strong structural changes induced by $\gamma$-irradiation. An analogous behaviour was reported for the effect of heat denaturation on RNAse A [11].

As regards sulfur-containing residues, exposure of the protein even at the lowest irradiation dose produces significant changes in the $\mathrm{C}-\mathrm{S}$ bonds of the Met residues, as indicated by the splitting of the $724 \mathrm{~cm}^{-1}$ band (731 and $725 \mathrm{~cm}^{-1}$ ), as well as in Cys (Fig. 1). In fact, the four disulfide bridges of which two (Cys26-Cys84 and Cys58-Cys110) connect $\alpha$ - and $\beta$-strands, gave rise to a broad Raman band at about $515 \mathrm{~cm}^{-1}$, that sensitively changes its spectral features after irradiation (Fig. 1). The curve fitting analysis of the $\nu \mathrm{S}-\mathrm{S}$ region revealed that the protein structure, initially assuming mainly a gauche-gauche-gauche ( $\mathrm{ggg}$ ) configuration of the $\mathrm{C}_{\beta}-\mathrm{S}-\mathrm{S}-\mathrm{C}_{\beta^{\prime}}$ disulfide bridges $(>90 \%)$, takes also a gauche-gauche-trans (ggt) conformation (15\%) after 23 Gy irradiation that becomes the predominant form ( $>50 \%$ ) at 84 Gy (Fig. 3). Modifications of the S-S conformations were also confirmed by the intensity increase of the band at $749 \mathrm{~cm}^{-1}$ due to $\nu \mathrm{C}-\mathrm{S}$ of Cys (Fig. 1). The conformational S-S changes indicate that some disulfide bonds can be reductively opened, as observed in the reductive reactions of RNAse A by some reagents such as $\beta$-mercaptoethanol, and successively, at least in part, reoxidised contributing to the protein structure. The disulfide opening can be correlated with the radical-induced damages on Tyr, Met and the secondary structure changes. In fact, it is worth noting that two of the "buried" Tyr probably attacked by $\mathrm{H}^{\bullet}$ atoms, i.e. Tyr- 25 and Tyr- 97 which is strongly paired with Asp-83, are located near to the 26-84 disulfide bridge. In addition, since the bridge connects $\alpha$ - and $\beta$-strands, its opening induces the decrease of the $\alpha$-helix content which exposes two of the four Met residues (-29 and -30) sited in that $\alpha$-helical stretch (coordinates were taken from the Protein Data Bank, 5rsa structure).

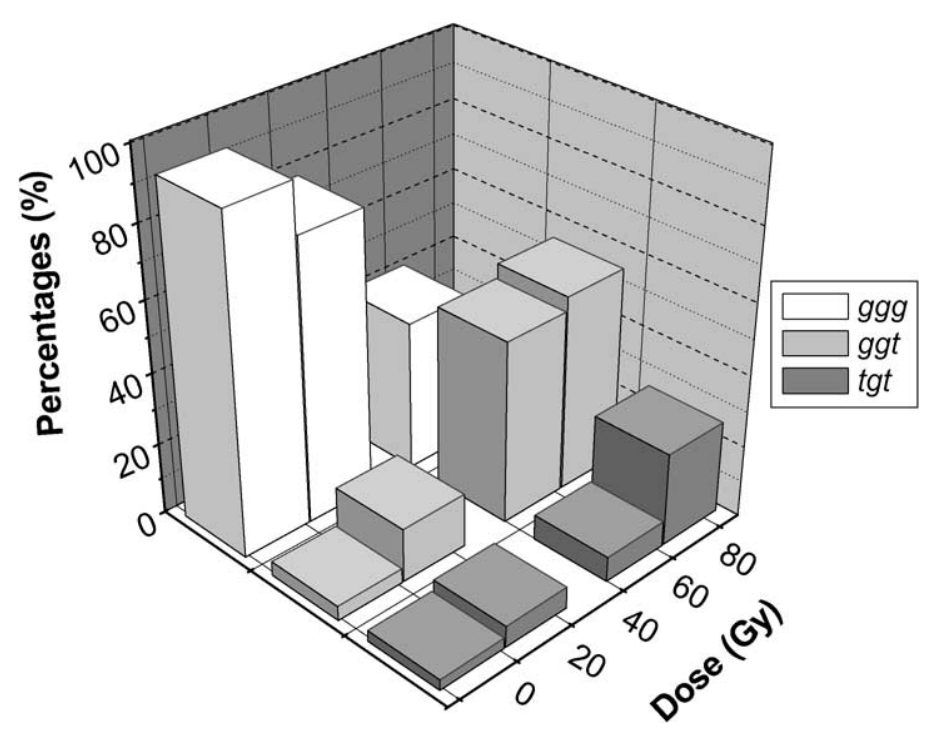

Fig. 3. Disulfide bridge conformations found in the $\mathrm{N}_{2} \mathrm{O}$-saturated $t$-BuOH-containing RNAse A samples after different irradiation exposures and relative block diagram. The percentages were calculated by the analysis of the $500-540 \mathrm{~cm}^{-1} \mathrm{Ra}^{-}$ man regions. ggg, ggt, tgt stand for gauche-gauche-gauche, gauche-gauche-trans, trans-gauche-trans conformations of the $\mathrm{C}_{\beta}-\mathrm{S}-\mathrm{S}-\mathrm{C}_{\beta}$ conformations. 
As the irradiation dose was increased, changes were still visible both in the $\nu \mathrm{C}-\mathrm{S}$ bands of Met and Cys, indicating that the action of $\mathrm{H}^{\bullet}$ atoms gives rise to a profound change in the sulfur-containing residues. These results well agree with the selective attack by $\mathrm{H}^{\bullet}$ atoms on Cys and Met reported in the literature [12,13], leading to changes in the primary structure of the protein. In fact, the $\mathrm{H}^{\bullet}$-induced desulfurisation involving these residues yield $\alpha$-aminobutyrric acid (Abu) and Ala (reactions (5) and (6)). In addition, the reductive radical attack towards Met residues has been recently found to yield diffusible sulfur radicals able inducing damages in cellular membranes [4-6].

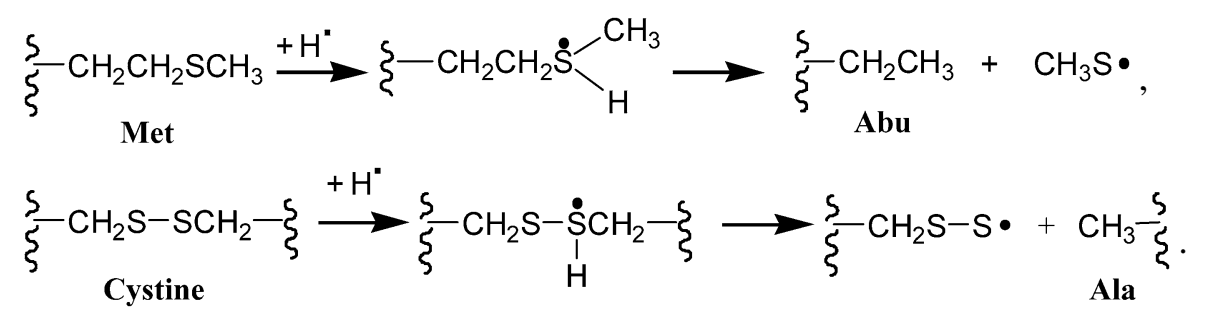

With respect to other possible reactive sites, the appearance of the weak band at $1587 \mathrm{~cm}^{-1}$ after only $23 \mathrm{~Gy}$ irradiation, due to $\nu \mathrm{C}_{4}=\mathrm{C}_{5}$ of the His imidazole ring, suggests the reductive radical attack involves also His, differently to that observed under oxidative stress conditions. Since His-12 and His-119 play an important role in the active site, this result explains the high ability in inactivating the enzyme reported in the literature [10]. Moreover, the attack on these residues can be connected to the changes observed in the Met and Tyr band since both Met-13 and Asp-14 H-bonded to Tyr-25 are located near His-12.

In conclusion, the reductive-radical attack seems to destabilise progressively the protein structure. In fact, prior to a complete unfolding transition, significant structural changes take place in some specific stretches of the amino acid sequence that are near in the 3D protein structure (i.e. 12-14, 25-30, 83-84, 119-120, etc.), while the overall backbone topology is still quite preserved. An analogous behaviour has been observed during thermal unfolding of RNAse A, where the complete unfolding comes after a pre-transition where the interaction of an $\alpha$-strand with the hydrophobic core is loosened, providing increased conformational freedom to the side chains involved in the interface [14].

To better mimic the biological situation, the protein was irradiated also in Ar-deaerated phosphate buffer solution. The presence of phosphate buffer as the medium at physiological $\mathrm{pH}$ (7.2) is also useful for increasing the reducing species concentration. In fact, under these experimental conditions a part of the solvated electrons will react with $\mathrm{H}_{2} \mathrm{PO}_{4}{ }^{-}$leading to $\mathrm{H}^{\bullet}$ atoms; thus, the reducing species present under these experimental conditions are two, hydrated electron and $\mathrm{H}^{\bullet}$ atom, having the radiation chemical yield of 2.16 and $0.83 \mu \mathrm{molj}^{-1}$, respectively. Unfortunately, the presence of the phosphate buffer in the samples makes no possible a deep analysis of some spectral regions, such as $1640-1670,870-1100$ and $500-570 \mathrm{~cm}^{-1}$.

As observed in the absence of buffer, exposure of the protein at the lowest irradiation dose was enough to cause significant changes in the sulfur moieties of RNAse A. In fact, the spectral modification visible in the bands due to the $\mathrm{C}-\mathrm{S}$ stretching mode of cystines and Met (751 and $729-723 \mathrm{~cm}^{-1}$, respectively) were very similar to those observed in RNAse A aqueous solution, further confirming that these residues are the preferential site of a reductive radical attack (Fig. 2(b) and (c)).

Changes in the strength of hydrogen bonding by Tyr residues were indicated by $I_{850} / I_{830}$ intensity ratio that increased of a less extent $(\approx 15 \%)$ than that observed in aqueous solution. This difference can 
be attributed to the presence of the buffer in the sample that can stabilise the protein structure rendering Tyr residues in the primary step more resistant towards radical attack than that in pure water.

In addition, the strong intensity increase of the band at $621 \mathrm{~cm}^{-1}$, due to Phe residues, indicated that the attack at the side chain of these residues takes place only when the reducing species concentration is enough high (Fig. 2(c)).

In conclusion, the results obtained in the presence also of hydrated electrons are very similar to those obtained with only $\mathrm{H}^{\bullet}$ atoms. This fact is in agreement with the literature where the action of $\mathrm{e}_{\mathrm{aq}}{ }^{-}$is reported to be essentially similar to that of $\mathrm{H}^{\bullet}$ atoms rather than of ${ }^{\bullet} \mathrm{OH}$ radicals and is concentrated on sulfur-containing and aromatic amino acids [15].

\section{Conclusions}

The sensitivity of some amino acid residues towards reducing radical attack is highlighted by the present results. Sulfur-containing residues (Met and cystine) and Tyr residues are the preferential sites when RNAse A undergoes reducing radical stress, analogously to that observed under oxidative conditions. At high doses there is some effect on the aromatic Phe residues, indicating the affinity of reducing species also in reacting with these residues, although both $\mathrm{e}_{\mathrm{aq}}{ }^{-}$and $\mathrm{H}^{\bullet}$ atoms will react in the primary step with other residues. Reductive stress causes changes in the primary protein structure as well as in secondary and tertiary structure. Progressive unfolding of the helical structure due to the rupture of disulfide bridges and the loss of intra-molecular Tyr $\mathrm{H}$-bonds indicate a higher denaturating ability of $\mathrm{H}^{\bullet}$ than that of $\mathrm{OH}^{\bullet}$ radicals. In conclusion, the results of $\gamma$-irradiation experiments reported in this paper can give a contribution to the knowledge of free radical-induced modifications in protein structure, enlarging the number of protein modifications detectable as result of exposure to radical stress conditions.

\section{References}

[1] E.R. Stadtman, Role of oxidised amino acids in protein breakdown and stability, Methods Enzymol. 258 (1995), $379-393$.

[2] C.L. Hawkins and M.J. Davies, Generation and propagation of radical reactions on proteins, Biochim. Biophys. Acta 1504 (2001), 196-219.

[3] W.M. Garrison, Reaction mechanisms in the radiolysis of peptides, polypeptides, and proteins, Chem. Rev. 87 (1987), 381-398.

[4] A. Torreggiani, M. Tamba, I. Manco, M.R. Faraone-Mennella, C. Ferreri and C. Chatgilialoglu, Investigation of radicalbased damage of RNAse A in aqueous solutions and lipid vesicles, Biopolymers 81 (2006), 39-50.

[5] C. Ferreri, I. Manco, M.R. Faraone-Mennella, A. Torreggiani, M. Tamba, S. Manara and C. Chatgilialoglu, The reaction of hydrogen atoms with methionine residues: a model of reductive radical stress causing tandem protein-lipid damage, Chem. BioChem. 7 (2006), 1738-1744.

[6] A. Torreggiani, M. Tamba, I. Manco, M.R. Faraone-Mennella, C. Ferreri and C. Chatgilialoglu, Radiation damage of lysozyme in a biomimetic model: some insights by Raman spectroscopy, J. Mol. Struct. 767 (2005), 744-747.

[7] N.N. Lichtin, J. Ogdan and G. Stein, Fast consecutive radical processes within the ribonuclease molecule in aqueous solution. II. Reaction with OH radicals and hydrated electrons, Biochim. Biophys. Acta 276 (1972), 124-142.

[8] A.P.J. Alix, G. Pedanou and M. Berjot, Fast determination of the quantitative secondary structure of proteins by using some parameters of Raman amide I band, J. Mol. Struct. 174 (1988), 159-164.

[9] A. Torreggiani, G. Bottura and G. Fini, Interaction of biotin and biotinyl derivatives with avidin: conformational changes upon binding, J. Raman Spectrosc. 31 (2000), 445-450.

[10] L.K. Mee, S.J. Adelstein and G. Stein, Inactivation of ribonuclease by the primary aqueous radicals, Radiat. Res. 52 (1972), 588-602.

[11] M.C. Chen and R.C. Lord, Laser Raman spectroscopic studies of the thermal unfolding of ribonuclease A, Biochemistry 15 (1976), 1889-1897. 
[12] R. Shapira and G. Stein, Reactions of aromatic and sulfur amino acids in ribonuclease with hydrogen atoms in water solution, Science 162 (1968), 1489-1491.

[13] B.E. Holmes, G. Navon and G. Stein, Action of atomic hydrogen on ribonuclease in aqueous solution, Nature 18 (1967), 1087-1091.

[14] A. Navon, V. Ittah, J.H. Laity, H.A. Scheraga, E. Haas and E.E. Gussakovsky, Local and long-range interactions in the thermal unfolding transition of bovine pancreatic ribonuclease A, Biochemistry $\mathbf{4 0}$ (2001), 93-104.

[15] G. Stein, The inactivation of some enzymes in aqueous solution by atomic hydrogen, in: Energetic and Mechanisms in Radiation Biology, G.O. Phillips, ed., Academic Press, London, 1968, pp. 467-477. 


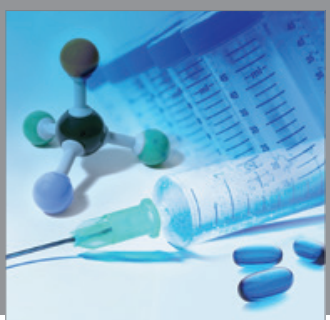

International Journal of

Medicinal Chemistry

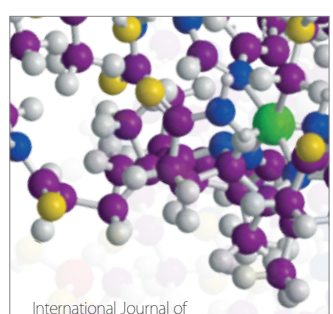

Carbohydrate Chemistry

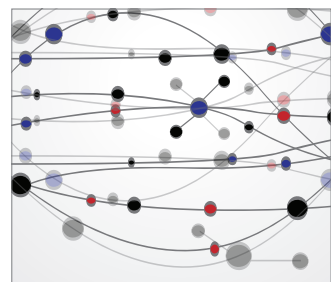

The Scientific World Journal
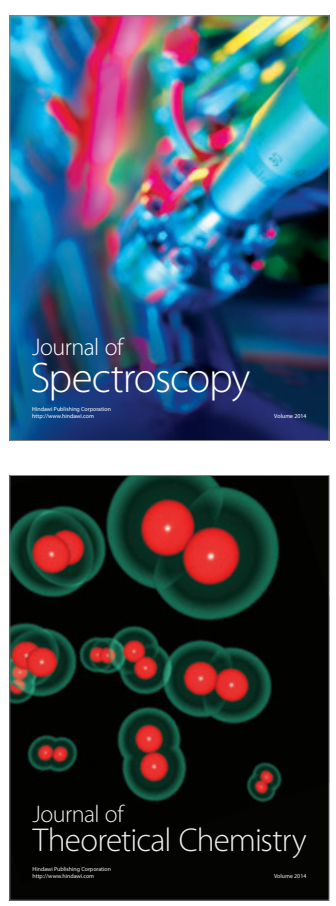
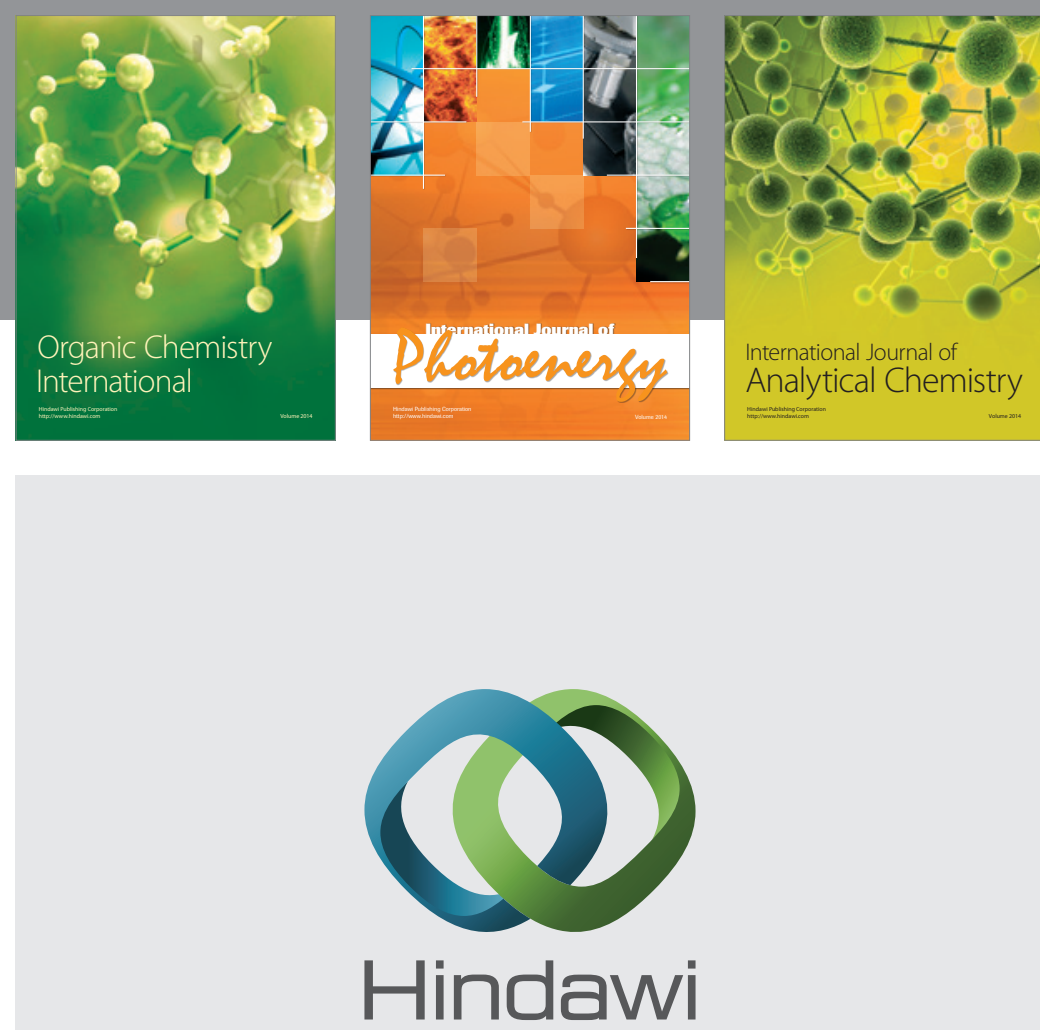

Submit your manuscripts at

http://www.hindawi.com
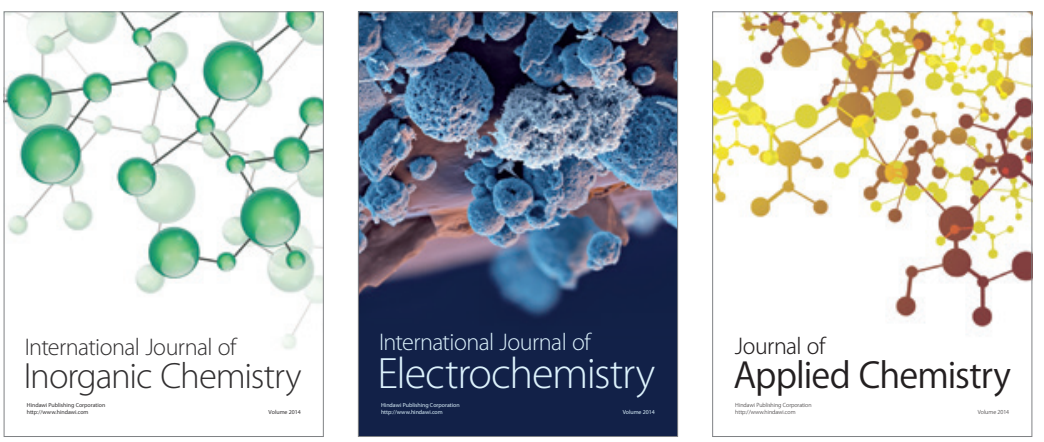

Journal of

Applied Chemistry
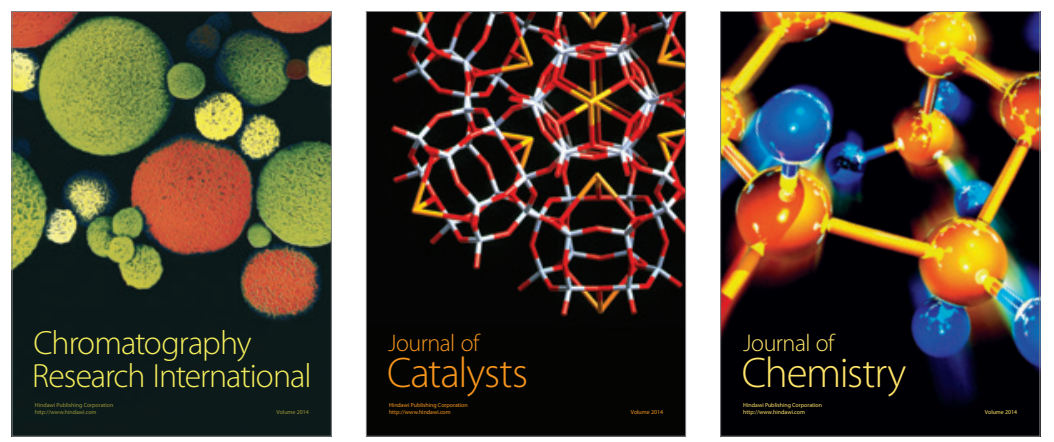
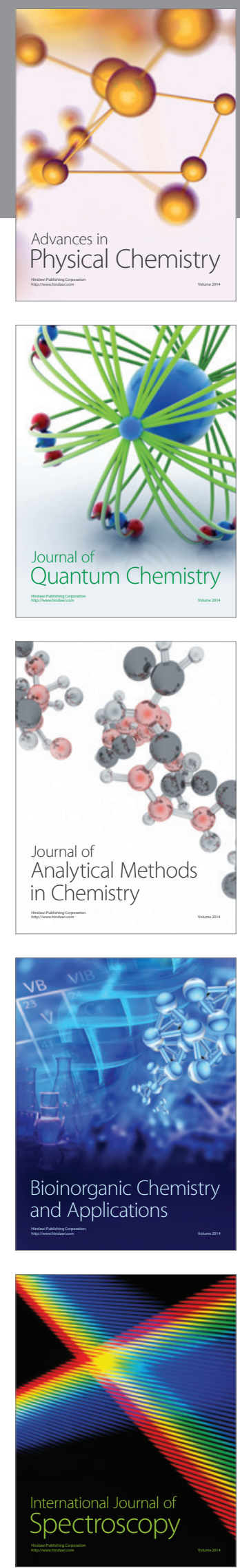